\title{
Carrier Phase Dependence in the Ionization of Rydberg Atoms by Short Radio-Frequency Pulses: A Model System for High Order Harmonic Generation
}

\author{
A. Gürtler, ${ }^{1}$ F. Robicheaux, ${ }^{3}$ M. J. J. Vrakking, ${ }^{1}$ W. J. van der Zande, ${ }^{1,2}$ and L. D. Noordam ${ }^{1}$ \\ ${ }^{1}$ FOM Institute for Atomic and Molecular Physics, Kruislaan 407, 1098SJ Amsterdam, The Netherlands \\ ${ }^{2}$ Department of Molecular and Laser Physics, University of Nijmegen, 6500GL Nijmegen, The Netherlands \\ ${ }^{3}$ Department of Physics, Auburn University, 206 Allison Lab, Auburn, Alabama 36849, USA
}

(Received 1 April 2003; published 10 February 2004)

\begin{abstract}
We report time-resolved electron emission in experiments on ionization of rubidium Rydberg atoms $(n=90)$ by few-cycle radio-frequency (RF) (1-10 MHz) pulses. The electron emission occurs in multiple bursts and strongly depends on the carrier-envelope phase as well as the duration and amplitude of the RF pulses. Remarkably, ionization is observed during a series of cycles with the same amplitude. Even at the low RF frequencies, ionization is not completed in a single cycle. Remixing of the states at the zero crossing of the field is believed to play an essential role. Similarities with the ionization process leading to high order harmonic generation are discussed.
\end{abstract}

DOI: $10.1103 /$ PhysRevLett.92.063901

PACS numbers: 42.65.Ky, 32.80.Rm

Ionization of atoms by electromagnetic radiation is known to depend strongly on the amplitude and frequency of the driving field. In most cases, the role of the phase of the carrier frequency is considered to be irrelevant. Recent calculations [1-3] and experiments $[4,5]$ show that, for ionization by short pulses containing only a few field cycles, the carrier phase does play an important role. Figure 1(a) shows a field $F(t)$ given by $F(t)=E(t) \sin (\omega t+\phi)$ with a Gaussian envelope $E(t)$ containing only two cycles at FWHM. As can be seen in the figure, a phase shift $\Delta \phi$ of $\frac{\pi}{2}$ changes the peak value of the central oscillation near the envelope maximum considerably. Moreover, the peak field is reached twice for $\phi=0$, but only once for $\phi=\frac{\pi}{2}$. Hence, for (nonlinear) processes in which the yield depends strongly on the peak value of the field, the carrier phase of the short pulse can be more important than the frequency. One such process is the emission of high order harmonics by noble gas atoms upon exposure to intense short optical pulses [4,7-10]. In this Letter, we describe experiments on a strongly related carrier phase-dependent process: ionization of highly excited Rydberg atoms by a short radiofrequency $(\mathrm{RF})$ pulse. In the experiments, we monitored the electron emission with subcycle time resolution and found that the emission pattern and total yield strongly depend on the carrier phase of the driving field and that a mechanism exists to spread the emission of electrons in bursts over many RF cycles [see, e.g., Fig. 1(b)]. It is remarkable that the ionization occurs in bursts even for high-amplitude pulses with a rectangular envelope, i.e., with subsequent half cycles of constant amplitude as shown in Fig. 2. We discuss the mechanism for this spreaded ionization. Finally, the impact of the observed subcycle features for the ionization process leading to high order harmonic generation (HHG) is discussed.

The experiments are performed in a vacuum chamber $\left(10^{-7} \mathrm{mbar}\right)$. An atomic $\mathrm{Rb}$ beam from a resistively heated oven crosses a nanosecond dye laser beam. The laser excites atoms in a two-photon process $(\lambda=593.72 \mathrm{~nm}$, $\Delta \lambda=0.005 \mathrm{~nm}$ ) to a high Rydberg state, typically $n=$ $90 d$. Two capacitor plates, on which the RF field is applied, surround the interaction region. Subsequent to the laser excitation, the atoms are exposed to the fewcycle RF pulse. The RF pulses are synthesized in a

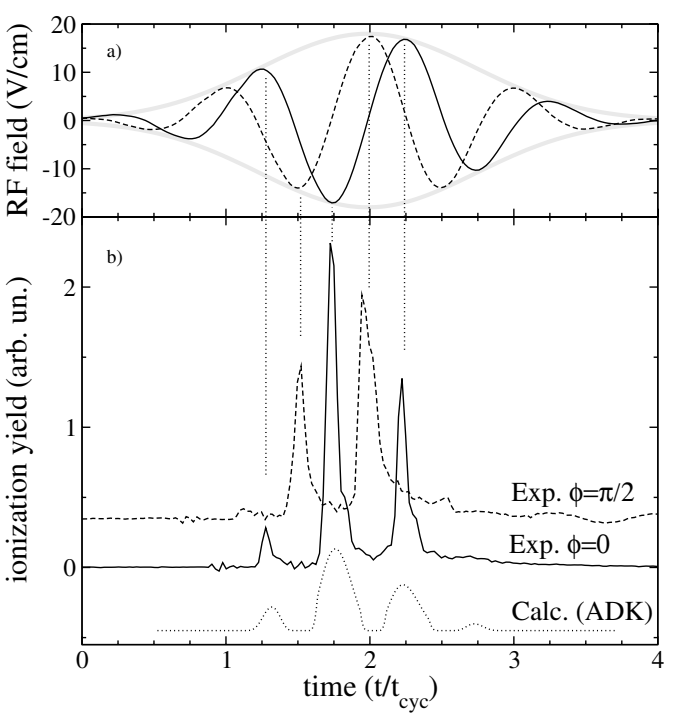

FIG. 1. (a) RF pulses with $6.25 \mathrm{MHz}$ carrier frequency and carrier-envelope phases of 0 (solid line) and $\frac{\pi}{2}$ (dashed line) as a function of time in units of the cycle time $t_{\mathrm{cyc}}=160 \mathrm{~ns}$. (b) Solid and dashed lines: Experimental ionization yield of a Rb $90 d$ state exposed to the RF pulses shown in (a). For comparison, the dotted line shows the ionization yield of atomic hydrogen subjected to a $10^{15} \mathrm{~W} / \mathrm{cm}^{2}$ optical pulse with the same field as the $\phi=0^{\circ}$ trace (solid line) calculated with an Ammosov-Delone-Krainov formula amended by an empirical expression to include barrier-suppression ionization [6]. 


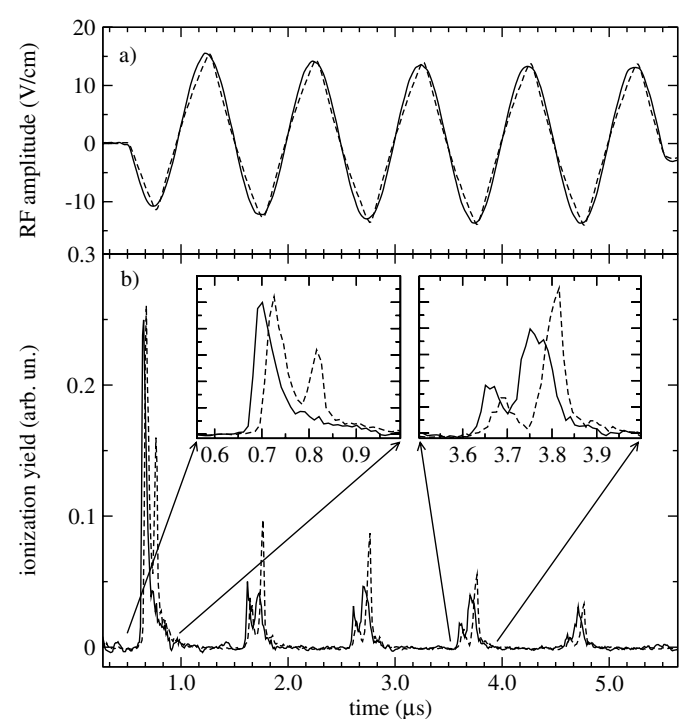

FIG. 2. (a) $1 \mathrm{MHz}$ RF pulseforms with sine (solid line) and triangular (dashed line) shapes. (b) Electron emission traces of a $90 d$ state subjected to the RF pulse forms shown in (a). Only the ionization by the negative part of the RF is shown.

programmable pulse generator, amplified by a RF amplifier, and sent to the capacitor plates using a transmission line configuration. Electrons released by the ionization process are accelerated by the RF field towards the detector. Electrons released at negative half cycles of the RF pulses are pushed towards the detector and recorded with a fast digital oscilloscope, while electrons released at positive cycles are pulled away from the detector. A complete picture of the ionization is obtained by adding two time traces with opposite field polarities. For traces similar to the ones shown in Fig. 1(b), we typically averaged over 100 laser shots. The microchannel plate detector has a time resolution of $3 \mathrm{~ns}$, which is much faster than the RF cycle period (100 ns at $10 \mathrm{MHz})$. The flight time from the interaction region to the detector is about $50 \mathrm{~ns}$, and corrected for by time shifting the electron emission traces. The flight-time dispersion due to different accelerating fields and differences in the starting positions of the electrons is about $15 \mathrm{~ns}$ and determines the time resolution of the experiment. Bound Rydberg state population, not ionized by the RF pulse, is probed afterwards by means of state-selective field ionization. To that end, the field on one of the capacitor plates is ramped in $3.5 \mu \mathrm{s}$ to $22 \mathrm{~V} / \mathrm{cm}$. In the time between the laser excitation and the onset of the RF pulse (typically $300 \mathrm{~ns}$ ), some $m$ mixing of the initially excited $m=0$ state by stray electric and magnetic fields can occur.

Figure 2(b) shows the time-resolved ionization of a $\mathrm{Rb}$ $90 d$ Rydberg state subjected to five-cycle sinusoidal (solid line) and triangular (dashed line) $1 \mathrm{MHz}$ RF pulses in a rectangular envelope as shown in Fig. 2(a). The emission occurs in bursts and is not depleted after the five-cycle pulse. Furthermore, the ionization peaks show a splitting which depends on the exact shape of the carrier wave (sine vs triangular) as well as the amplitude.

We further investigate why the electron emission is not depleted after the first cycle and the origin of the doublepeak structure. Figure 3(a) shows the state-selective field ionization trace of the initial $90 d$ state, i.e., the timedependent electron emission upon application of a slowly ramped electric field $\left(\frac{d F}{d t} \approx 6 \frac{\mathrm{V}}{\mathrm{cm} \mu \mathrm{s}}\right)$. In an electric field, the degenerate high- $\ell$ states split up in Stark states. The trace shows two features which result from different pathways while traversing the many crossings of Stark states in the time-dependent field from zero field to the ionization limit. The first, narrow peak comes from pathways which are mostly adiabatic, while the second, broad feature is attributed to mixed adiabatic-diabatic ionization and characteristic for field ionization of high angular momentum states (the electric field mixes the angular momenta) [11]. Integration of the total yield gives the ionized fraction at a particular peak-field strength as shown in Fig. 3(b). The ionization yield is clearly not a step as a function of the peak-field amplitude. This indicates that, if in an RF experiment the peak amplitude of a cycle is somewhere on the rising edge of the curve in Fig. 3(b), the Rydberg atom population is only partly ionized during that cycle. During the cycle the field lowers again and changes sign, and the angular momentum of the remaining bound state fraction of the wave function will remix. Such remixing is a nonhydrogenic core effect
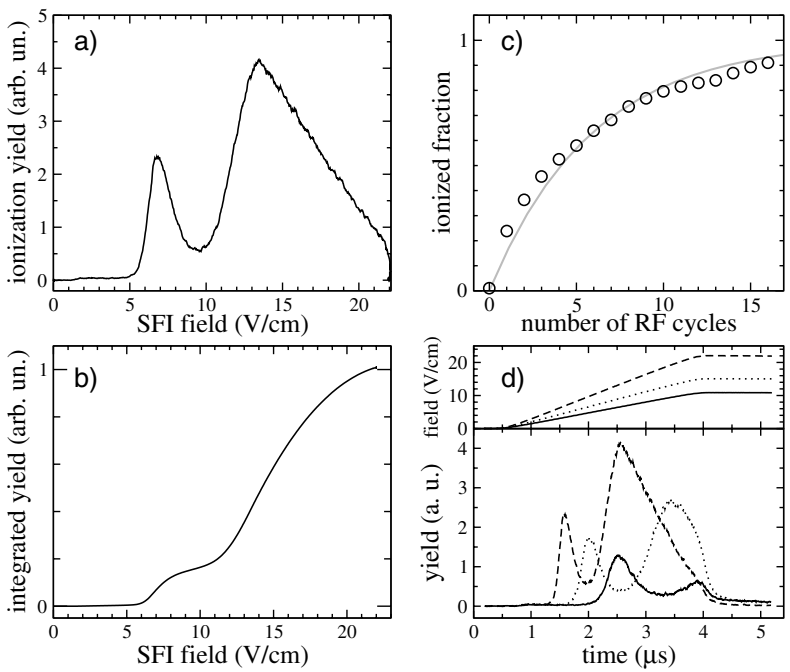

FIG. 3. (a) Field ionization yield of the $\mathrm{Rb} 90 \mathrm{~d}$ state by a slowly ramped $\left(22 \mathrm{~V} \mathrm{~cm}^{-1} / 3.5 \mu \mathrm{s}\right)$ electric field. (b) Integral of (a). (c) Ionized fraction of the Rydberg population as a function of the number of cycles in a $10 \mathrm{MHz}, 13.5 \mathrm{~V} / \mathrm{cm}$ pulse with a rectangular envelope. The line represents an exponential fit to the data. Each subsequent cycle ionizes $\approx 20 \%$ of the remaining Rydberg population. (d) Upper panel: Field ramps applied to ionize the $90 d$ state. Lower panel: Time dependence of the electron emission for the field ramps shown in the upper panel. 


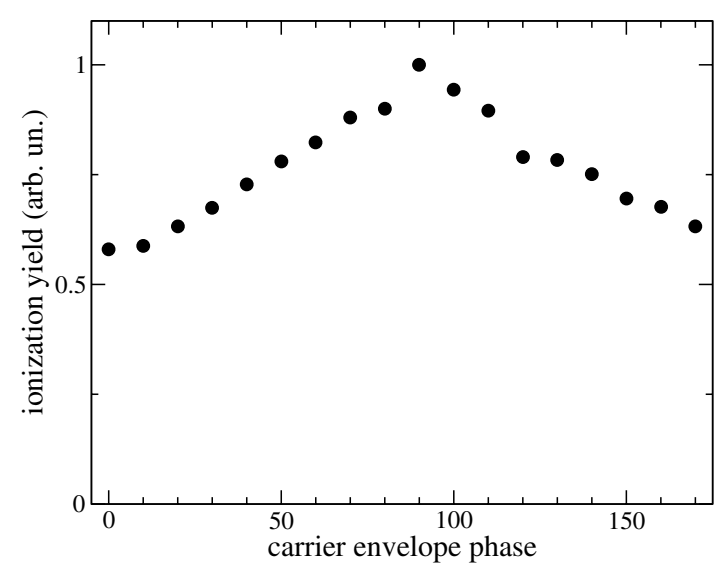

FIG. 4. Total ionization yield as a function of carrierenvelope phase for a $90 d$ state subjected to a RF pulse with $6 \mathrm{MHz}$ carrier frequency and an envelope amplitude of $14.6 \mathrm{~V} / \mathrm{cm}$. The initial $90 d$ state is not depleted after the pulse with a duration of two cycles at FWHM.

of the Rb Stark states. At the peak amplitude of the subsequent half cycle again a fraction will be ionized. As a result, it takes a few cycles to ionize the Rydberg atom population completely. This is explicitly demonstrated in Fig. 3(c), which shows the ionization yield as a function of the number of cycles in a rectangular pulse envelope together with an exponential fit. Mahon et al. observed already that long pulse RF ionization of Rydberg atoms require quasistatic field amplitudes [12]. Figure 3(c) refines this picture: Even at these strong fields it takes many cycles until the initial state is depleted. Figure 3(d) shows the field ionization process in greater detail. The lower panel shows the time-dependent emission upon application of electric field ramps which rise in $3.5 \mu$ s to $22 \mathrm{~V} / \mathrm{cm}$ (dashed line), $15 \mathrm{~V} / \mathrm{cm}$ (dotted line), and $11 \mathrm{~V} / \mathrm{cm}$ (solid line). It can be clearly seen that, once the maximum field value is reached, the ionization rate drops sharply, even if not all the Rydberg population is ionized. Without remixing by the RF field, the remaining population is stable.

It has been predicted that the total ionization yield of atoms subjected to few-cycle laser pulses depends on the carrier-envelope (CE) phase [2]. To our knowledge, this is not yet confirmed by experiments. Figure 4 shows that this effect can be easily observed in the radio-frequency domain. The figure shows the integrated ionization yield of a $\mathrm{Rb} 90 d$ state subjected to a $6 \mathrm{MHz} \mathrm{RF}$ pulse as a function of carrier-envelope phase. The envelope amplitude is constant $(14.6 \mathrm{~V} / \mathrm{cm})$ and the pulse energy in this scan rises about $11 \%$ from $0^{\circ}$ to $90^{\circ}$ carrier phase. The ionization yield increases almost a factor of 2 if the carrier phase is near $90^{\circ}$ and the cycle amplitude is at the peak of the envelope.

The exact timing of the electron emission is believed to play an essential role in the field of HHG. The classical HHG model introduced by Corkum in 1993 [13] starts by ionization of the atom by a strong optical laser field. The ionization is considered to take place near the maxima of the optical field cycles by tunneling ionization. The quasifree electron wave packets are pushed back to the ionic core when the optical field changes sign after about $10^{-15} \mathrm{~s}$. If emitted at the proper phase, the electron revisits the ionic core with a considerable energy gained from the optical field. Then the electron may recombine to the ground state and this energy is released as a vacuum ultraviolet or even extreme ultraviolet (XUV) photon. This XUV emission process can take place during every half cycle of the optical pulse. The total emission pattern therefore consists of a train of attosecond XUV bursts, rather than a single attosecond pulse [7,14], unless the driving pulse reaches the few-cycle regime [4]. The tunneling ionization rate strongly depends on the maximum field strength during the cycle, and for few-cycle pulses the electron ejection rate and, hence, the XUV emission yield depend on the carrier phase of the optical pulses $[1,3]$. In the optical domain, the electron emission, the initial step of HHG, has never been observed with a subcycle resolution. Such observations are frustrated by the high frequency of optical pulses. This constraint is relaxed by about 8 orders of magnitude in the case of RF ionization of Rydberg atoms, as reported here. Note that although the characteristic time scales and length scales of ground-state and Rydberg atoms are very different, the electron makes many round-trips per cycle of the field; hence, the electron samples the slowly varying potential in many orbits. The photon energy is much smaller than the binding energy in both cases, such that many photons are involved in the ionization process [15].

For further comparison of optical ground-state and RF Rydberg ionization, we return to Fig. 1(b), which shows the measured time-resolved electron emission of $\mathrm{Rb} n=$ $90 d$ states exposed to a $6.25 \mathrm{MHz}$ pulse of $1 \mu$ s duration (about two cycles at FWHM). The emission is compared for two different carrier phases (solid line: $\phi=0$, dashed line: $\phi=\frac{\pi}{2}$ ) of the RF field. The integrated power of the pulses is equal within 3\%. In both cases, the initial Rydberg population is depleted at the end of the pulses. In HHG, the highest energy electrons are made when the cycle amplitude is maximal and thus photons with the highest XUV photon energy are produced. In Fig. 1(b), the electron emission in the strongest half cycle is phase dependent, explaining why HHG is observed to depend on the CE phase [4].

In HHG, the highest energy electrons are made when the cycle amplitude is maximal and thus photons with the highest XUV photon energy are produced. The experiments shown in Fig. 5 demonstrate why short pulses are advantageous for the generation of high orders of harmonics [10]. The two electron emission traces in Fig. 5(b) are recorded with two RF pulses $(10 \mathrm{MHz}$, peak amplitude $21.5 \mathrm{~V} / \mathrm{cm}$ ) which have a factor 12 different pulse duration (95 ns, and $1.2 \mu \mathrm{s}$, respectively). For the short 


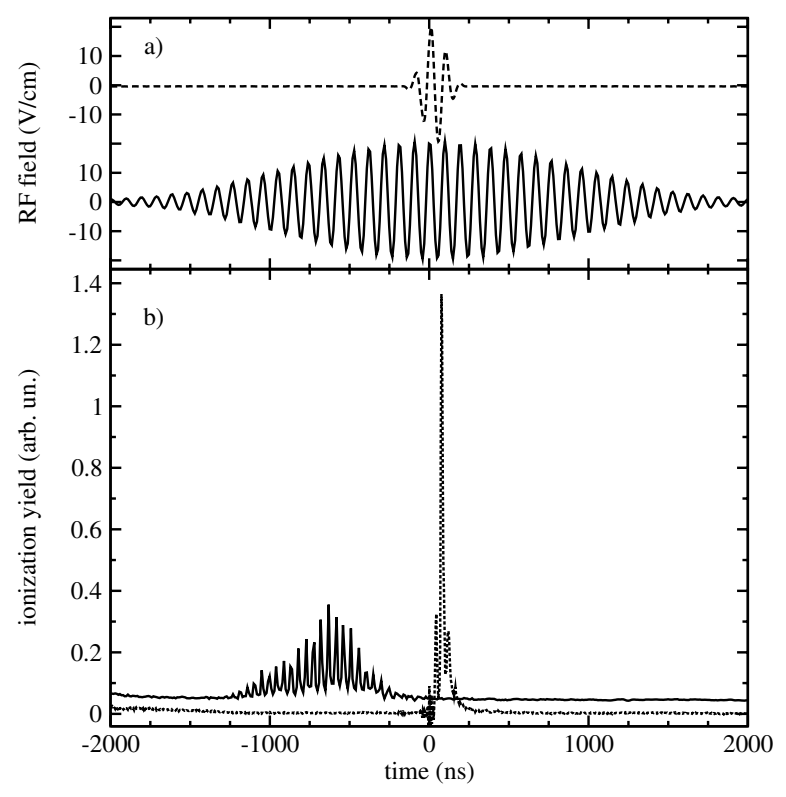

FIG. 5. (a) $10 \mathrm{MHz}$ RF pulses with equal amplitudes of $21.5 \mathrm{~V} / \mathrm{cm}$ and durations of $95 \mathrm{~ns}$ (dashed line) respectively $1.2 \mu \mathrm{s}$ (solid line). b) Electron emission of a $90 d$ state subjected to the RF pulses in (a). The traces are set off vertically for clarity.

pulse, the population is not yet depleted when the peak of the pulse is reached and a considerable fraction of the electrons is ejected at the peak of the pulse. For long pulses, the population is depleted well before the peak is reached [16].

In contrast to the optical ionization of ground-state atoms, the RF ionization of Rydberg atoms involves the traversal of many avoided crossings of Stark states, which can be traversed diabatically or adiabatically. In general, this traversal is completed in a mixed diabatic-adiabatic process. The resulting wave function is a (incoherent) superposition of Stark states with different energies and ionization rates, leading to the observed broad threshold. In the optical case, the number of states involved is much smaller. For very short optical pulses, nonadiabatic effects have been seen, and it has been shown that the contribution of excited bound states is not negligible and leads to an ionization suppression similar to the one seen in our experiment [10].

In summary, we observed that the electron emission of Rydberg atoms exposed to few-cycle RF pulses occurs in multiple bursts. The actual pulse parameters (amplitude, frequency, and phase) determine the number and relative height of the emission peaks as well as the field value at which the maximum emission occurs. The investigated system displays many similarities with the physics under- lying high order harmonic generation and is believed to be an ideal model system. Detailed understanding of the observed double-peak phenomenon in Fig. 2 requires further experiments and theoretical modeling and can contribute to the discussion over the optimal pulse shape for HHG. An interesting question is how different pulse shapes can be employed to optimize a desired ionization behavior $[4,17,18]$.

A.G. acknowledges financial support from the European Community's Human Potential Programme under Contract No. HPRN-CT-1999-00129, COCOMO. F. R. was supported by the NSF. This work is part of the research program of the "Stichting voor Fundamenteel Onderzoek der Materie (FOM)," which is financially supported by the "Nederlandse Organisatie voor Wetenschappelijk Onderzoek (NWO)."

[1] A. de Bohan, P. Antoine, D. B. Milošević, and B. Piraux, Phys. Rev. Lett. 81, 1837 (1998).

[2] I. P. Christov, Opt. Lett. 24, 1425 (1999).

[3] G. Tempea, M. Geissler, and T. Brabec, J. Opt. Soc. Am. B 16, 669 (1999).

[4] A. Baltuška et al., Nature (London) 421, 611 (2003).

[5] G. G. Paulus, F. Grasbon, H. Walther, P. Villoresi, M. Nisoli, S. Stagira, E. Priori, and S. de Silvestri, Nature (London) 414, 182 (2001).

[6] D. Bauer and P. Mulser, Phys. Rev. A 59, 569 (1999).

[7] P. M. Paul, E. S. Toma, P. Breger, G. Mullot, F. Augé, P. Balcou, H. G. Muller, and P. Agostini, Science 292, 1689 (2001).

[8] B. Sheehy, J. D. D. Martin, L. F. DiMauro, P. Agostini, K. J. Schafer, M. B. Gaarde, and K. C. Kulander, Phys. Rev. Lett. 83, 5270 (1999).

[9] A. LHuillier and P. Balcou, Phys. Rev. Lett. 70, 774 (1993).

[10] I. P. Christov, J. Zhou, J. Peatross, A. Rundquist, M. M. Murnane, and H. C. Kapteyn, Phys. Rev. Lett. 77, 1743 (1996).

[11] T. F. Gallagher, Rydberg Atoms (Cambridge University Press, Cambridge, England, 1994).

[12] C. R. Mahon, J. L. Dexter, P. Pillet, and T. F. Gallagher, Phys. Rev. A 44, 1859 (1991).

[13] P. B. Corkum, Phys. Rev. Lett. 71, 1994 (1993).

[14] P. Antoine, A. LHuillier, and M. Lewenstein, Phys. Rev. Lett. 77, 1234 (1996).

[15] B. E. Sauer, S. Yoakum, L. Moorman, P. M. Koch, D. Richards, and P. A. Dando, Phys. Rev. Lett. 68, 468 (1992).

[16] P. Lambropoulos, Phys. Rev. Lett. 55, 2141 (1985).

[17] A. M. Weiner, Rev. Sci. Instrum. 71, 1929 (2000).

[18] T. Brixner, N. H. Damrauer, P. Niklaus, and G. Gerber, Nature (London) 414, 57 (2001). 